COMMENT. In an editorial, Hirsch LJ of Yale University Epilepsy Center notes that the mortality of status epilepticus is 15 to $22 \%$ and the outcome correlates with seizure duration. (N Engl J Med 2012;366:659-660). The definition of status has been shortened from 30 minutes to 5 to 10 minutes in recent studies. Seizures lasting $>5$ minutes are likely to be self-sustained and require intervention. The RAMPAR Trial reported above found that the more rapid administration of the IM midazolam (1.2 min) than the IV lorazepam (4.8 $\mathrm{min})$ outweighed the faster cessation of seizures with intravenous administration (1.6 min IV vs $3.3 \mathrm{~min}$ via IM route). The rate of hospitalization was lower in the IM-midazolam group, as compared with the IVlorazepam group (57.6\% vs 65.6\%). Dr Hirsch comments that home treatment for status epilepticus may be found more satisfactory in the future, using the nasal or buccal routes for administering midazolam.

\title{
COMBINATION DRUG THERAPY IN REFRACTORY EPILEPSY
}

Researchers from University of Washington, Seattle, WA analyzed the treatment records from 148 developmentally disabled adults with refractory epilepsy cared for in 2 state-run institutions. Records charted monthly convulsive seizure occurrence and AED regimen over 30 years. Patients had a predominance of focal over generalized EEG abnormalities. The effects of 8 commonly used AEDs alone and in combination on seizure frequency were studied in within-patient comparisons. In decreasing order of frequency, the drugs compared were lamotrigine, valproate, carbamazepine, phenytoin, topiramate, levetiracetam, gabapentin, and zonisamide; phenobarbital and oxcarbazine were used at only one institution and were excluded from calculations. Individual AED combinations were first compared to an aggregate measure of all other combinations to which a patient had been exposed. This allowed greater statistical power to assess efficacy of individual combinations.

Out of the most frequently used AED combinations, only lamotrigine (LTG) and valproate (VPA) combination had superior efficacy; seizure frequency was reduced by $50 \%$ or more in comparison to other regimens. The LTG/VPA combination was superior to VPA or LTG monotherapy, and CBZ/VPA, VPA/GBP, or CBZ/VPA/PHT combinations. While 2 concurrent AEDs provided improved efficacy over monotherapy, use of 3 AEDs at a time provided no further benefit over two AEDs combined. AEDs should be used no more than 2 at a time for optimal response. (Poolos NP, Warner LN, Humphreys SZ, Williams S. Comparative efficacy of combination drug therapy in refractory epilepsy. Neurology Jan 3, 2012;78:62-68). (Response and reprints: Dr Poolos. E-mail:npoolos@uw.edu).

COMMENT. The superior efficacy of LTG/VPA combination compared to other AED combinations is reported previously (Brodie MJ et al. Epilepsy Res 1997;26:423432)(Pisani F et al. Epilepsia 1999;40:1141-1146) (Refs cited by authors). Synergism between the 2 drugs may explain the improved seizure control by a valproate-induced reduction in hepatic clearance of lamotrigine. However, the present study found no VPA effect on LTG serum concentrations, and the mechanism of synergism is unexplained. 